Federal Reserve Bank of Dallas

Globalization and Monetary Policy Institute

Working Paper No. 243

http://www.dallasfed.org/assets/documents/institute/wpapers/2015/0243.pdf

\title{
On the Sustainability of Exchange Rate Target Zones with Central Parity Realignments*
}

\author{
Enrique Martínez-García \\ Federal Reserve Bank of Dallas
}

June 2015

\begin{abstract}
I show that parity realignments alone do not suffice to ensure the long-run sustainability of an exchange rate target zone with imperfect credibility due to the gambler's ruin problem. However, low credibility and frequent realignments can destabilize the exchange rate.
\end{abstract}

JEL codes: E58, F31, F33, F41, G15

\footnotetext{
* Enrique Martínez-García, Federal Reserve Bank of Dallas, 2200 N. Pearl Street, Dallas, TX 75201. 214-922-5262. enrique.martinez-garcia@dal.frb.org. https://sites.google.com/site/emg07uw. The views in this paper are those of the author and do not necessarily reflect the views of the Federal Reserve Bank of Dallas or the Federal Reserve System.
} 


\section{Introduction}

Exchange rates exhibit persistent misalignments and excess volatility (Obstfeld and Rogoff (2000)). One strand of the literature argues that these empirical regularities may be an unintended consequence of monetary policy (Dornbusch (1976), Eichenbaum and Evans (1995), Scholl and Uhlig (2008)). From that perspective, researchers have long debated the (de)stabilizing role of Taylor-type monetary policy rules (Mark (2009), Clarida (2014)) and the relative (un)importance of monetary shocks (Benigno and Benigno (2008), Valcárcel (2013)) as factors driving the exchange rate.

Another strand of the literature has focused on the effects of different arrangements and interventions in the foreign exchange market (Portugal Duarte et al. (2011)). Krugman's (1991) seminal contribution on this topic suggests that exchange rate volatility can be lower in a credible target zone than under free-floating. Recurring currency pressures on emerging economies and increased financial volatility have contributed since 2008 to a global shift toward managing exchange rates aimed at attaining greater stability (IMF (2014)). ${ }^{1}$

A large body of theoretical work pioneered by Bertola and Caballero (1992a,b) recognizes that exchange rate commitments are generally not backed in all circumstances (imperfect credibility) and incorporates a moving parity into the target zone model. In that setting, low credibility with frequent parity realignments can lead to more volatile exchange rates than free-floating - unlike in Krugman's (1991) fully credible target zone. $^{2}$ However, the vulnerabilities of a target zone with imperfect credibility have received less attention (Delgado and Dumas (1993), Cornell (2003)).

This paper focuses on the role of imperfect credibility in the long-run sustainability of a target zone. I confirm that target zones with limited credibility induce higher exchange rate volatility than free-floating. I also show that the exchange rate hits the intervention bands of the target zone in finite time irrespective of the frequency with which the band is realigned. Furthermore, even though such a target zone is defended only occasionally after hitting the bands, I prove that an eventual collapse cannot be ruled out as any finite amount of foreign reserves will be exhausted in finite time almost surely. Hence, I conclude that a target zone with parity realignments can be destabilizing and remains vulnerable over the long-run.

\section{The Monetary Model}

I consider a two-country model with complete asset markets and frictionless goods markets, where purchasingpower parity $(P P P)$ and the uncovered interest parity condition $(U I P)$ hold. The description of the economy is completed with a pair of equations arising from the investment-savings $\left(I S^{R}\right)$ and money demand $\left(M M^{R}\right)$ behavior across countries. The continuous-time model of nominal exchange rate determination can be stated

\footnotetext{
${ }^{1}$ Countries with soft pegs (including conventional pegged arrangements, pegged exchange rates within horizontal bands, crawling pegs, stabilized arrangements, and crawl-like arrangements) accounted for 43.5 percent of all IMF members in 2014 (IMF (2014)).

${ }^{2}$ Bertola and Svensson (1993), Tristani (1994) and Werner (1995) provide extensions and an early empirical assessment of target zones with imperfect credibility. Portugal Duarte et al. (2011) survey the more recent literature.
} 
$\operatorname{as}^{3}$

$$
\begin{aligned}
\mathbf{I S}^{\mathbf{R}} & : \quad \frac{\mathbb{E}_{t}\left(d c^{R}(t)\right)}{d t}=\frac{1}{\gamma}\left[i^{R}(t)-\frac{\mathbb{E}_{t}\left(d p_{t}^{R}\right)}{d t}\right], \\
\mathbf{M M}^{\mathbf{R}} & : i^{R}(t)=\gamma\left(\frac{1-\beta}{\beta}\right)\left[c^{R}(t)+p_{t}^{R}-f(t)\right], \\
\mathbf{P P P} & : \quad s(t)=p^{R}(t), \\
\mathbf{U I P} & : \frac{\mathbb{E}_{t}(d s(t))}{d t}=i^{R}(t) .
\end{aligned}
$$

I denote $c^{R}(t)$ as the per capita consumption differential across countries, $p^{R}(t)$ as the price differential, $i^{R}(t)$ as the interest rate differential, and $s(t)$ as the nominal exchange rate.

The monetary fundamentals are given by $f(t)=m^{R}(t)+v(t)$, where $m^{R}(t)$ denotes the money supply differential across countries and $v(t)$ represents nonsterilized interventions in the foreign exchange market. I model $m^{R}(t)$ with the following driftless diffusion:

$$
d m^{R}(t)=\kappa d W(t), m^{R}(0)=0,
$$

where $W(t)$ is a standard Brownian motion.

A solution to the system in (1) - (4) reduces to a standard present-value equation for the nominal exchange rate,

$$
s(t)=f(t)+\frac{1}{\gamma}\left(\frac{\beta}{1-\beta}\right) \frac{\mathbb{E}_{t}(d s(t))}{d t},
$$

and full consumption equalization across countries-i.e., $c^{R}(t)=0$.

\section{The Target Zone with Imperfect Credibility}

The exchange rate is a time-invariant-potentially nonlinear - function of the fundamentals:

Proposition 1 The family of solutions for the system (5) - (6) is

$$
s(t)=f(t)+B\left[e^{-\lambda f(t)}-e^{\lambda f(t)}\right]
$$

where $\lambda=\sqrt[2]{2 \frac{\gamma}{\kappa^{2}}\left(\frac{1-\beta}{\beta}\right)}$.

A target zone with imperfect credibility is described with a symmetric intervention band over the fundamentals, centered around $f_{c}(t)$ with a constant width of $2 \bar{f}$. When the lower margin of the band is reached at time $t$, with probability $(1-\delta)$ a defense is mounted and the fundamentals return in a discrete jump to the midpoint of the existing band $f_{c-}(t) \equiv \lim _{\alpha \rightarrow 0} f_{c}(t-\alpha)$. With probability $0<\delta<1$, the band is realigned keeping its width unchanged. The lower margin of the abandoned band $f_{c-}(t)-\bar{f}$ becomes the upper margin

\footnotetext{
${ }^{3}$ All these equilibrium conditions are $\log$-linearized. The parameter $\gamma$ is the coefficient of relative risk aversion, and $\beta$ the discount rate.
} 
of the new band, and fundamentals are set in a discrete jump at the midpoint of the new band $f_{c-}(t)-2 \bar{f}$. If fundamentals reach the upper margin, an analogous set of possibilities describes the intervention choices. ${ }^{4}$

The central parity starts at zero $\left(f_{c}(0)=0\right)$ and is constant almost everywhere, except on a measure-zero set of time periods when a realignment occurs. All interventions to defend or realign the band after hitting its margins result in jumps of absolute size $\bar{f}$ that change foreign reserves. ${ }^{5}$ The nonsterilized intervention process $v(t)$ is given by

$$
v(t)=j(t) \bar{f}, v(0)=0
$$

where $j(t)$ is a jump process constant almost everywhere except on a measure-zero set of time periods when the intervention margins are reached. The process $j(t)$ counts the net number of interventions (positive minus negative) in the interval $[0, t]$.

Corollary 1 In a free-floating regime, $B=0$ and the exchange rate in (7) implies $s(t)=f(t)=m^{R}(t)$. In a target zone with imperfect credibility, the coefficient $B$ in (7) is given by

$$
B \equiv-e^{\lambda f_{c-}(t)} \bar{B}, \bar{B} \equiv-\left(\delta-\frac{1}{2}\right) \frac{\bar{f}}{\sinh (\lambda \bar{f})}
$$

If the target zone lacks sufficient credibility (i.e., if $\delta>\frac{1}{2}$ ), then $B<0$ and the exchange rate displays an inverted "S-curve."

Corollary 1 shows that a target zone with low credibility $\left(\delta>\frac{1}{2}\right)$ leads to more volatile exchange rates than free-floating. Corollary 1 also implies that the central parity on the exchange rate equals $f_{c-}(t)$ and the bands imposed around it by the target zone are uniquely identified as $\left[f_{c-}(t)-2 \delta \bar{f}, f_{c-}(t)+2 \delta \bar{f}\right]$. Hence, the width of the exchange rate bands relates to the width of the intervention band on fundamentals $(2 \bar{f})$ and the probability of realignment $(\delta)$.

I define the difference between the managed and the free-floating exchange rates as $q(t) \equiv s(t)-f(t)$. The dynamics of the exchange rate $s(t)$ can be expressed in terms of $q(t)$ as follows:

Corollary 2 If $\delta \neq \frac{1}{2}$, the exchange rate solution implied by (7) is given by

$$
\begin{aligned}
& d s(t)=\frac{1}{2} \kappa^{2} \lambda^{2} q(t) d t+\kappa\left[1-2 \lambda \bar{B} \sqrt[2]{1+\frac{1}{4 \bar{B}^{2}}} q(t)^{2}\right] d W(t), s(0)=f_{c}(0)=0, \\
& d q(t)=\frac{1}{2} \kappa^{2} \lambda^{2} q(t) d t-2 \kappa \lambda \bar{B} \sqrt[2]{1+\frac{1}{4 \bar{B}^{2}} q(t)^{2}} d W(t), q(0)=0 .
\end{aligned}
$$

The drift and volatility of $s(t)$ are (nonlinear) functions of $q(t)$. If $\delta=\frac{1}{2}$, the exchange rate solution is the same as under free-floating.

Corollary 2 points out that the target zone is isomorphic to an intervention band over the process $q(t)$.

\subsection{Properties of the Intervention Band}

The central result on the properties of the intervention band is as follows:

\footnotetext{
${ }^{4}$ The fully credible solution of Krugman (1991) arises in the limit as $\delta \rightarrow 0$.

${ }^{5}$ Exchange rate jumps are not expected ex ante to rule out arbitrage opportunities.
} 
Proposition 2 Let $f(t)$ within the intervention band be given by the 1-dimensional diffusion process in (5). Define the stopping time $\tau$ as the first time fundamentals hit the intervention band

$$
\tau=\inf \left\{t \geq 0: f(t) \notin\left(f_{c-}(t)-\bar{f}, f_{c-}(t)+\bar{f}\right)\right\} .
$$

Then, it follows that the probability of reaching the upper (lower) margin conditional on hitting the band, denoted $p \equiv P_{f_{c-}(\tau)}\left(f(\tau)=f_{c-}(\tau)+\bar{f}\right)\left(1-p \equiv P_{f_{c-}(\tau)}\left(f(\tau)=f_{c-}(\tau)-\bar{f}\right)\right)$, is

$$
p=\frac{1}{2}
$$

The expected duration of the non-intervention period is

$$
\mathbb{E}_{f_{c-}(\tau)}[\tau]=\left(\frac{\bar{f}}{\kappa}\right)^{2}
$$

which depends on the width of the band $(2 \bar{f})$ and the volatility of the fundamentals $(\kappa)$ alone.

Given the assumption of a symmetric band and driftless fundamentals, the probability of hitting either margin of the intervention band starting at the midpoint is $\frac{1}{2}$. Proposition 2 also shows that the time interval between interventions is finite almost surely and has an expected duration given by (14). Therefore, it follows that the probability of a parity realignment $(\delta)$ does not affect the odds of reaching the upper margin $(p)$ or the duration of each non-intervention spell.

\subsection{Target Zone Sustainability}

Regardless of the realignment probability $\delta$, Proposition 2 implies that both net changes in foreign reserves through nonsterilized interventions $v(t)=j(t) \bar{f}$ and the process $j(t)$ that tracks the net number of interventions follow a generalized random walk with variable transition probabilities and random time steps. The process $j(t)$ increases by one with a gain of $\bar{f}$ reserves whenever there is either an upward realignment after hitting the upper margin or an upward intervention to defend the band after reaching the lower margin, and it decreases by one with a loss of $\bar{f}$ reserves in all other circumstances when reaching the margins of the band.

A variant of the gambler's ruin problem in statistics (Degroot (1975)) arises whereby any attempt to use nonsterilized purchases/sales of foreign reserves by domestic policymakers to manage the exchange rate becomes almost surely unsustainable with finite reserves over the long run: ${ }^{6}$

Proposition 3 In a target zone with imperfect credibility, net changes in foreign reserves $v(t)$ through nonsterilized interventions of size $0<\bar{f}<\infty$ also change the stock of money. An initial amount of foreign reserves $0<H<\infty$ sets an implicit lower bound on the finite stock of money. Exhausting foreign reserves forces the target zone to be abandoned. Given Proposition 2, the probability of gaining (losing) reserves every time the margins of the band are reached is $\frac{1}{2}$. Furthermore, the probability of depleting all available reserves $H$ leading to the eventual collapse of the target zone is 1 whenever this regime is expected to remain indefinitely.

\footnotetext{
${ }^{6} \mathrm{I}$ assume the domestic country bears alone the burden of managing the target zone. Accordingly, $v(t)$ captures the nonsterilized change in foreign reserves held by domestic policymakers that are part of the domestic stock of money.
} 
It follows from Proposition 3 that nonsterilized discrete interventions must eventually exhaust any finite amount of foreign reserves irrespective of the probability of realigning the band $\delta$. Hence, I conclude that central parity realignments do not necessarily prevent the eventual collapse of a target zone with imperfect credibility. In turn, as noted in Corollary 1, a target zone with low credibility may exacerbate the volatility of exchange rates.

\section{Concluding Remarks}

In this paper, I present a standard exchange rate determination model under an imperfectly credible target zone. Discrete nonsterilized interventions using finite foreign reserves are undertaken occasionally to defend the exchange rate bands, but parity realignments occur occasionally. From this stylized framework, I reach two main conclusions. First, central parity realignments do not necessarily eliminate the gambler's ruin problem. Therefore, the long-run sustainability of a target zone cannot be guaranteed. Second, the probability of losing foreign reserves can be independent of the probability of parity realignment. Furthermore, the odds are that those losses will exhaust any finite amount of foreign reserves almost surely causing the

target zone to be abandoned. Hence, parity realignments alone do not rule out the target zone's eventual collapse, but with low credibility such an arrangement can contribute to increased exchange rate instability.

Acknowledgement I thank Charles Engel, Sabrina Güttes, Rodolfo Manuelli and an anonymous referee for their helpful advice and suggestions, and Valerie Grossman for excellent research assistance. An online appendix with all the proofs can be found at: https://sites.google.com/site/emg0\%uw/. All remaining errors are mine alone.

\section{References}

[1] Benigno, G. and P. Benigno, 2008, Exchange Rate Determination Under Interest Rate Rules. Journal of International Money and Finance 27(6), 971-999.

[2] Bertola, G. and R. J. Caballero, 1992a, Target Zones and Realignments. American Economic Review $82(3), 520-536$.

[3] Bertola, G. and R. J. Caballero, 1992b, Sustainable Intervention Policies and Exchange Rate Dynamics, in Exchange Rate Targets and Currency Bands. (P. Krugman and M. Miller (eds.), Cambridge University Press, New York).

[4] Bertola, G. and L.E.O. Svensson, 1993, Stochastic Devaluation Risk and the Empirical Fit of TargetZone Models. Review of Economic Studies 60(3), 689-712.

[5] Clarida, R., 2014, Monetary Policy in Open Economies: Practical Perspective for Pragmatic Central Bankers. Journal of Economic Dynamics and Control 49, 21-30.

[6] Cornell, C. M., 2003, Target Zones, Reserve Crises, and Inverted S-Curves. Journal of International Financial Markets, Institutions and Money 13(4), 313-323.

[7] Degroot, M. H., 1975, Probability and Statistics. (Addison-Wesley, Reading, Massachusetts).

[8] Delgado, F. and B. Dumas, 1993, Monetary Contracting Between Central Banks and the Design of Sustainable Exchange-Rate Zones. Journal of International Economics 34(3-4), 201-224. 
[9] Dornbusch, R., 1976, Expectations and Exchange Rate Dynamics. Journal of Political Economy 84(6), 1161-1176.

[10] Eichenbaum, M. and C. Evans, 1995, Some Empirical Evidence of Shocks to Monetary Policy on Exchange Rates. Quarterly Journal of Economics 110(4), 975-1010.

[11] Harris, J. W. and H. Stocker, 1998, Handbook of Mathematics and Computational Science. (Springer, New York).

[12] IMF, 2014, Annual Report on Exchange Arrangements and Exchange Restrictions. (Washington, October 2014).

[13] Karatzas, I. and S. E. Shreve, 1988, Brownian Motion and Stochastic Calculus. (Springer-Verlag, New York).

[14] Krugman, P. R., 1991, Target Zones and Exchange Rate Dynamics. Quarterly Journal of Economics $106,669-682$.

[15] Mark, N. C., 2001, International Macroeconomics and Finance: Theory and Econometric Methods. (Blackwell Publishers Inc., Malden, Massachusetts).

[16] Mark, N. C., 2009, Changing Monetary Policy Rules, Learning and Real Exchange Dynamics. Journal of Money, Credit and Banking 41(6), 1047-1070.

[17] Obstfeld, M. and K. Rogoff, 2000, The Six Major Puzzles in International Macroeconomics: Is There a Common Cause? in NBER Macroeconomics Annual 2000, Vol. 15, 339-412. (B. Bernanke and K. Rogoff (eds.), MIT Press, Cambridge, Massachusetts).

[18] Portugal Duarte, A., J. Sousa Andrade and A. Duarte, 2011, Exchange Rate Target Zones: A Survey of the Literature. Journal of Economic Surveys 27, 247-268.

[19] Scholl, A. and H. Uhlig, 2008, New Evidence on the Puzzles: Results from Agnostic Identification on Monetary Policy and Exchange Rates. Journal of International Economics 76(1), 1-13.

[20] Tristani, O., 1994, Variable Probability of Realignment in a Target Zone. Scandinavian Journal of Economics 96(1), 1-14.

[21] Valcárcel, V., 2013, Exchange Rate Volatility and the Time-varying Effects of Aggregate Shocks. Journal of International Money and Finance 32, 822-843.

[22] Werner, A. M., 1995, Exchange Rate Target Zones, Realignments and the Interest Rate Differential: Theory and Evidence. Journal of International Economics 39(3), 353-367. 


\section{APPENDIX}

\section{A Properties of a Regulated Process ${ }^{7}$}

The properties of a regulated process studied in this Appendix apply to any well-behaved diffusion process, not just to the particular application discussed in the body of the paper.

Theorem 1 Let me assume the process y $(t)$ can be represented by a 1-dimensional diffusion process of the form

$$
d y(t)=\eta(y(t)) d t+\sigma(y(t)) d W(t), y(0)=y_{0},
$$

which satisfies the Lipschitz conditions, i.e.,

$$
\begin{aligned}
|\eta(x)-\eta(z)|+|\sigma(x)-\sigma(z)| & \leq L|x-z|, \\
|\eta(x)|+|\sigma(x)| & \leq L(1+|x|) .
\end{aligned}
$$

Let me define the generator $L$ associated with this diffusion as

$$
L=\eta(y) \frac{\partial}{\partial y}+\frac{1}{2} \sigma^{2}(y) \frac{\partial^{2}}{\partial y^{2}},
$$

and the stopping time $\tau$ which denotes the first time the process hits the intervention band as

$$
\tau=\inf \{t \geq 0: y(t) \notin(\underline{y}, \bar{y})\} .
$$

Then, given the initial condition $y_{0} \in(\underline{y}, \bar{y})$, it follows that:

(a) The process $y(t)$ hits the margins of the intervention band in finite time almost surely, i.e.,

$$
\tau<\infty P_{y_{0}}-\text { a.s. }
$$

if there exists a function $h \in C^{2}$ which satisfies that $\operatorname{Lh}(y)=1$.

(b) The probability of reaching the upper margin conditional on hitting the band is denoted $p \equiv P_{y_{0}}(y(\tau)=\bar{y})$. This probability can be computed as

$$
p \equiv P_{y_{0}}(y(\tau)=\bar{y})=\frac{k\left(y_{0}\right)-k(\underline{y})}{k(\bar{y})-k(\underline{y})},
$$

where the (non-constant) function $k \in C^{2}$ satisfies that $L k(y)=0$. Furthermore, it follows from (16) that the probability of reaching the lower margin conditional on hitting the band is $P_{y_{0}}(y(\tau)=\underline{y}) \equiv 1-p$.

(c) The expected time interval until the process $y(t)$ hits the margins of the zone can be measured as

$$
\mathbb{E}_{y_{0}}[\tau]=p h(\bar{y})+(1-p) h(\underline{y})-h\left(y_{0}\right),
$$

which is a function of $h, k \in C^{2}$, the initial condition $y_{0}$ and the margins of the band $(\underline{y}, \bar{y})$.

Proof of Theorem 1. Let me assume that there exist time-invariant functions $h, k \in C^{2}$ such that $L h(y)=1$ and $L k(y)=0$. Using Itô's lemma, I say that

$$
\begin{aligned}
d h(y) & =h^{\prime}(y) \sigma(y) d W+L h(y) d t \\
& =h^{\prime}(y) \sigma(y) d W+d t \\
d k(y) & =k^{\prime}(y) \sigma(y) d W+L k(y) d t \\
& =k^{\prime}(y) \sigma(y) d W .
\end{aligned}
$$

\footnotetext{
${ }^{7}$ For further detailes, see Karatzas and Shreve (1988).
} 
Integrating both sides, I get that

$$
\begin{aligned}
& h(y(t))=h(y(0))+\int_{0}^{t} h^{\prime}(y(u)) \sigma(y(u)) d W(u)+t, \\
& k(y(t))=k(y(0))+\int_{0}^{t} k^{\prime}(y(u)) \sigma(y(u)) d W(u),
\end{aligned}
$$

where $M_{h}(t) \equiv \int_{0}^{t} h^{\prime}(y(u)) \sigma(y(u)) d W(u)$ and $M_{k}(t) \equiv \int_{0}^{t} k^{\prime}(y(u)) \sigma(y(u)) d W(u)$ are two local martingales. Let me compute $M_{h}(t \wedge \tau)$ and $M_{k}(t \wedge \tau)$ as

$$
\begin{aligned}
& M_{h}(t \wedge \tau) \equiv \int_{0}^{t} \theta_{h}(u) d W(u), \theta_{h}(u) \equiv 1_{[0, \tau]}(u) h^{\prime}(y(u)) \sigma(y(u)), \\
& M_{k}(t \wedge \tau) \equiv \int_{0}^{t} \theta_{k}(u) d W(u), \theta_{k}(u) \equiv 1_{[0, \tau]}(u) k^{\prime}(y(u)) \sigma(y(u)),
\end{aligned}
$$

which are also local martingales. Since the interval $[y, \bar{y}]$ is bounded and $\sigma(y)$ satisfies the Lipschitz conditions, it must be the case that $C_{h} \equiv \sup \left|h^{\prime}(y) \sigma(y)\right|<\infty$ and $C_{k} \equiv \sup \left|k^{\prime}(y) \sigma(y)\right|<\infty$. So, it follows that

$$
[\underline{y}, \bar{y}] \quad[\underline{y}, \bar{y}]
$$

$$
\begin{aligned}
& \mathbb{E} \int_{0}^{t} \theta_{h}(u)^{2} d u \leq \mathbb{E} \int_{0}^{t}\left(\sup _{[\underline{y}, \bar{y}]}\left|h^{\prime}(y) \sigma(y)\right|\right)^{2} d u \leq C_{h}^{2} t<\infty, \\
& \mathbb{E} \int_{0}^{t} \theta_{k}(u)^{2} d u \leq \mathbb{E} \int_{0}^{t}\left(\sup _{[\underline{y}, \bar{y}]}\left|k^{\prime}(y) \sigma(y)\right|\right)^{2} d u \leq C_{k}^{2} t<\infty .
\end{aligned}
$$

This proves that $M_{h}(t \wedge \tau)$ and $M_{k}(t \wedge \tau)$ are martingales, not just local martingales. Then, $\mathbb{E}\left[M_{h}(t \wedge \tau)\right]=$ $\mathbb{E}\left[M_{h}(0)\right]$ and $\mathbb{E}\left[M_{k}(t \wedge \tau)\right]=\mathbb{E}\left[M_{k}(0)\right]$ with $M_{h}(0)=M_{k}(0)=0$.

(a) Let me denote $\mathbb{E}_{y_{0}}[\cdot]$ the expectation conditional on $y(0)=y_{0} \in(\underline{y}, \bar{y})$ such that

$$
\mathbb{E}_{y_{0}}[h(y(t \wedge \tau))]=h\left(y_{0}\right)+\mathbb{E}_{y_{0}}[t \wedge \tau]
$$

Since the interval $[\underline{y}, \bar{y}]$ is bounded, it must be the case that $D_{h} \equiv \sup _{[\underline{y}, \bar{y}]}|h(y)|<\infty$. So, I argue that

$$
\begin{aligned}
\mathbb{E}_{y_{0}}[t \wedge \tau] & =\mathbb{E}_{y_{0}}[h(y(t \wedge \tau))]-h\left(y_{0}\right) \\
& \leq\left|h\left(y_{0}\right)\right|+\left|\mathbb{E}_{y_{0}}[h(y(t \wedge \tau))]\right| \leq 2 D_{h}<\infty
\end{aligned}
$$

Therefore, by the Monotone Convergence Theorem, $\mathbb{E}_{y_{0}}[\tau] \leq 2 D_{h}<\infty$ as $t \rightarrow \infty$. This result implies that $\tau<\infty P_{y_{0}}-$ a.s.

(b) Let me denote $p \equiv P_{y_{0}}(y(\tau)=\bar{y})$ as the probability of hitting the upper band conditional on starting at $y(0)=y_{0}$ and reaching the margins of the band. I compute the conditional expectation of $k(\cdot)$ as

$$
\mathbb{E}_{y_{0}}[k(y(t \wedge \tau))]=k\left(y_{0}\right) .
$$

Since the interval $[\underline{y}, \bar{y}]$ is bounded, it must be true that $D_{k} \equiv \sup _{[\underline{y}, \bar{y}]}|k(y)|<\infty$. Then, these two results hold:

$$
\begin{aligned}
|k(y(t \wedge \tau))| & \leq D \forall(t, \omega) \\
\lim _{t \rightarrow \infty} k(y(t \wedge \tau)) & =k(y(\tau)) \text { a.s. }
\end{aligned}
$$


where $\omega \in \Omega$ denotes an event of the probability space. Hence, by the Dominated Convergence Theorem,

$$
k\left(y_{0}\right)=\lim _{t \rightarrow \infty} \mathbb{E}_{y_{0}}[k(y(t \wedge \tau))]=\mathbb{E}_{y_{0}}[k(y(\tau))]=p k(\bar{y})+(1-p) k(\underline{y}) .
$$

So, it follows that $p \equiv P_{y_{0}}(y(\tau)=\bar{y})=\frac{k\left(y_{0}\right)-k(\underline{y})}{k(\bar{y})-k(\underline{y})}$. Furthermore, it follows that the probability of reaching the lower margin conditional on hitting the band is $P_{y_{0}}(y(\tau)=y) \equiv 1-p$.

(c) Based on the previous results and the Dominated Convergence Theorem it must be true that

$$
\begin{aligned}
\mathbb{E}_{y_{0}}[\tau] & =\lim _{t \rightarrow \infty} \mathbb{E}_{y_{0}}[t \wedge \tau]=\lim _{t \rightarrow \infty} \mathbb{E}_{y_{0}}[h(y(t \wedge \tau))]-h\left(y_{0}\right) \\
& =\mathbb{E}_{y_{0}}[h(y(\tau))]-h\left(y_{0}\right) .
\end{aligned}
$$

Moreover, I can compute the conditional expectation of $h(y(\tau))$ as $\mathbb{E}_{y_{0}}[h(y(\tau))]=p h(\bar{y})+(1-p) h(\underline{y})$. Hence, from here it follows that

$$
\mathbb{E}_{y_{0}}[\tau]=p h(\bar{y})+(1-p) h(\underline{y})-h\left(y_{0}\right),
$$

which is a function of $h, k \in C^{2}$, the initial condition $y_{0}$ and the margins of the band $(\underline{y}, \bar{y})$.

\section{B Proofs}

Proof of Proposition 1. I conjecture that $s(t) \equiv G(f(t))$, and use Itô's lemma to argue that

$$
\begin{aligned}
d s(t) & =G^{\prime}(f(t)) \kappa d W(t)+\frac{\kappa^{2}}{2} G^{\prime \prime}(f(t)) d t, \\
\frac{\mathbb{E}_{t}(d s(t))}{d t} & =\frac{\kappa^{2}}{2} G^{\prime \prime}(f(t)) .
\end{aligned}
$$

If I replace this expression in (6), I obtain a second-order linear differential equation in $G(\cdot)$,

$$
G^{\prime \prime}(f(t))-2 \frac{\gamma}{\kappa^{2}}\left(\frac{1-\beta}{\beta}\right) G(f(t))=-2 \frac{\gamma}{\kappa^{2}}\left(\frac{1-\beta}{\beta}\right) f(t)
$$

The solution of this ODE can be expressed as

$$
s(t) \equiv G(f(t))=f(t)+A e^{\lambda f(t)}+B e^{-\lambda f(t)},
$$

where $\lambda=\sqrt[2]{2 \frac{\gamma}{\kappa^{2}}\left(\frac{1-\beta}{\beta}\right)}$. Requiring that $G\left(f_{c}(t)\right)=f_{c}(t)$, it follows that $A=-B$.

Proof of Corollary 1. Ruling out bubbles, equation (6) can be integrated between $t$ and infinity to obtain

$$
s(t)=\gamma\left(\frac{1-\beta}{\beta}\right) \int_{t}^{\infty} \mathbb{E}_{t}(f(u)) e^{-\gamma\left(\frac{1-\beta}{\beta}\right)(u-t)} d u,
$$

which shows that the exchange rate is a function of discounted current and expected future fundamentals. Given equation (17), the no-bubbles solution for the exchange rate must satisfy that $s(t)=f(t)$. Hence, the free-floating exchange rate is a special case of (7) in Proposition 1 that requires $A=B=0$. Given that no interventions in the foreign exchange market are needed (i.e., $v(t)=0$ ), the solution becomes $s(t)=m^{R}(t)$. In other words, in a regime with no interventions in the foreign exchange markets, the nominal exchange rate should be equal to the money supply differential.

Given Proposition 1, a solution to the target zone with imperfect credibility must belong to the family described in (7). To determine $A$ and $B$ conditional on $f_{c-}(t) \equiv \lim _{\alpha \rightarrow 0} f_{c}(t-\alpha)$ and $\bar{f}$, I impose that the exchange rate at which the band is reached must be equal to the expected exchange rate given the probability 
of realignment - and the two possible outcomes: either the band is realigned or defended - to avoid jumps in the exchange rate. Hence, the following conditions must hold:

$$
\begin{aligned}
& G\left(f_{c-}(t)+\bar{f} \mid f_{c-}(t), \bar{f}\right)=\delta G\left(f_{c-}(t)+2 \bar{f} \mid f_{c-}(t)+2 \bar{f}, \bar{f}\right)+(1-\delta) G\left(f_{c-}(t) \mid f_{c-}(t), \bar{f}\right), \\
& G\left(f_{c-}(t)-\bar{f} \mid f_{c-}(t), \bar{f}\right)=\delta G\left(f_{c-}(t)-2 \bar{f} \mid f_{c-}(t)-2 \bar{f}, \bar{f}\right)+(1-\delta) G\left(f_{c-}(t) \mid f_{c-}(t), \bar{f}\right),
\end{aligned}
$$

for all $f_{c-}(t)$ and $\bar{f}$. Notice that $f_{c-}(t)$ represents the central parity before the process hits the margins of the band, and is itself a function of time. Using (7), I obtain that

$$
\begin{aligned}
& f_{c-}(t)+\bar{f}+A e^{\lambda\left(f_{c-}(t)+\bar{f}\right)}+B e^{-\lambda\left(f_{c-}(t)+\bar{f}\right)}=\delta\left(f_{c-}(t)+2 \bar{f}\right)+(1-\delta) f_{c-}(t), \\
& f_{c-}(t)-\bar{f}+A e^{\lambda\left(f_{c-}(t)-\bar{f}\right)}+B e^{-\lambda\left(f_{c-}(t)-\bar{f}\right)}=\delta\left(f_{c-}(t)-2 \bar{f}\right)+(1-\delta) f_{c-}(t),
\end{aligned}
$$

where the right-hand side follows from the fact that the nominal exchange rate must be equal to the fundamentals at the midpoint of the prevailing band.

After some algebra, I infer from these two conditions that

$$
\begin{aligned}
A & =\frac{(1-2 \delta) \bar{f}}{e^{\lambda\left(f_{c-}(t)-\bar{f}\right)}-e^{\lambda\left(f_{c-}(t)+\bar{f}\right)}}=e^{-\lambda f_{c-}(t)} \frac{(1-2 \delta) \bar{f}}{e^{-\lambda \bar{f}}-e^{\lambda \bar{f}}}=e^{-\lambda f_{c-}(t)}\left(\delta-\frac{1}{2}\right) \frac{\bar{f}}{\sinh (\lambda \bar{f})}, \\
B & =-\frac{(1-2 \delta) \bar{f}}{e^{-\lambda\left(f_{c-}(t)+\bar{f}\right)}-e^{-\lambda\left(f_{c-}(t)-\bar{f}\right)}}=-e^{\lambda f_{c-}(t)} \frac{(1-2 \delta) \bar{f}}{e^{-\lambda \bar{f}}-e^{\lambda \bar{f}}}=-e^{\lambda f_{c-}(t)}\left(\delta-\frac{1}{2}\right) \frac{\bar{f}}{\sinh (\lambda \bar{f})},
\end{aligned}
$$

where the last equality follows from the definition of the hyperbolic sine (Harris and Stocker, 1998, p. 247). Hence, the solution in (7) takes the special form,

$$
\begin{aligned}
s(t) & =f(t)-\frac{(1-2 \delta) \bar{f}}{e^{-\lambda \bar{f}}-e^{\lambda \bar{f}}}\left[e^{-\lambda\left(f(t)-f_{c-}(t)\right)}-e^{\lambda\left(f(t)-f_{c-}(t)\right)}\right] \\
& =f(t)-\left(\delta-\frac{1}{2}\right) \frac{\bar{f}}{\sinh (\lambda \bar{f})}\left[e^{-\lambda\left(f(t)-f_{c-}(t)\right)}-e^{\lambda\left(f(t)-f_{c-}(t)\right)}\right] .
\end{aligned}
$$

The intervention band over fundamentals is defined as $f(t) \in\left[f_{c-}(t)-\bar{f}, f_{c-}(t)+\bar{f}\right]$. It follows immediately that the central parity of the intervention band on the fundamentals is equal to the central parity of the exchange rate $f_{c-}(t)$. If fundamentals reach the upper margin $f_{c-}(t)+\bar{f}$, then I can compute the upper margin on the nominal exchange rate $\bar{s}$ as follows:

$$
\begin{aligned}
\bar{s}(t) & =f_{c-}(t)+\bar{f}-\left(\delta-\frac{1}{2}\right) \frac{\bar{f}}{\sinh (\lambda \bar{f})}\left[e^{-\lambda\left(f_{c-}(t)+\bar{f}-f_{c-}(t)\right)}-e^{\lambda\left(f_{c-}(t)+\bar{f}-f_{c-}(t)\right)}\right] \\
& =f_{c-}(t)+\bar{f}-2\left(\delta-\frac{1}{2}\right) \frac{\bar{f}}{\sinh (\lambda \bar{f})}\left[\frac{e^{-\lambda \bar{f}}-e^{\lambda \bar{f}}}{2}\right] \\
& =f_{c-}(t)+\bar{f}+2\left(\delta-\frac{1}{2}\right) \frac{\bar{f}}{\sinh (\lambda \bar{f})} \sinh (\lambda \bar{f}) \\
& =f_{c-}(t)+2 \delta \bar{f} .
\end{aligned}
$$

Similarly, if fundamentals reach the lower margin $f_{c-}(t)-\bar{f}$, then the lower margin on the exchange rate $\underline{s}$ 
is given by:

$$
\begin{aligned}
\bar{s}(t) & =f_{c-}(t)-\bar{f}-\left(\delta-\frac{1}{2}\right) \frac{\bar{f}}{\sinh (\lambda \bar{f})}\left[e^{-\lambda\left(f_{c-}(t)-\bar{f}-f_{c-}(t)\right)}-e^{\lambda\left(f_{c-}(t)-\bar{f}-f_{c-}(t)\right)}\right] \\
& =f_{c-}(t)-\bar{f}-2\left(\delta-\frac{1}{2}\right) \frac{\bar{f}}{\sinh (\lambda \bar{f})}\left[\frac{e^{\lambda \bar{f}}-e^{-\lambda \bar{f}}}{2}\right] \\
& =f_{c-}(t)-\bar{f}-2\left(\delta-\frac{1}{2}\right) \frac{\bar{f}}{\sinh (\lambda \bar{f})} \sinh (\lambda \bar{f}) \\
& =f_{c-}(t)-2 \delta \bar{f} .
\end{aligned}
$$

The margins and central parity for any exchange rate solution given by (7) under a target zone with imperfect credibility are uniquely identified by the intervention band over the fundamentals, i.e., the intervention band over fundamentals $f(t) \in\left[f_{c-}(t)-\bar{f}, f_{c-}(t)+\bar{f}\right]$ implies a target zone on the exchange rate of the following form: $s(t) \in\left[f_{c-}(t)-2 \delta \bar{f}, f_{c-}(t)+2 \delta \bar{f}\right]$.

Proof of Corollary 2. From Corollary 1, the nominal exchange rate can be expressed as

$$
\begin{aligned}
& s(t)=f(t)+\bar{B}\left[e^{-\lambda\left(f(t)-f_{c-}(t)\right)}-e^{\lambda\left(f(t)-f_{c-}(t)\right)}\right], \\
& q(t) \equiv s(t)-f(t)=\bar{B}\left[e^{-\lambda\left(f(t)-f_{c-}(t)\right)}-e^{\lambda\left(f(t)-f_{c-}(t)\right)}\right],
\end{aligned}
$$

where $f_{c-}(t)$ is constant within the intervention band and $\bar{B} \equiv-\left(\delta-\frac{1}{2}\right) \frac{\bar{f}}{\sinh (\lambda \bar{f})}$. Applying Itô's lemma to (7), I obtain

$$
\begin{aligned}
d s(t) & =\frac{1}{2} \kappa^{2}\left[\lambda^{2} \bar{B}\left(e^{-\lambda\left(f(t)-f_{c-}(t)\right)}-e^{\lambda\left(f(t)-f_{c-}(t)\right)}\right)\right] d t+\kappa\left[1-\lambda \bar{B}\left(e^{-\lambda\left(f(t)-f_{c-}(t)\right)}+e^{\lambda\left(f(t)-f_{c-}(t)\right)}\right)\right] d W(t) \\
d q(t) & =\frac{1}{2} \kappa^{2}\left[\lambda^{2} \bar{B}\left(e^{-\lambda\left(f(t)-f_{c-}(t)\right)}-e^{\lambda\left(f(t)-f_{c-}(t)\right)}\right)\right] d t-\kappa\left[\lambda \bar{B}\left(e^{-\lambda\left(f(t)-f_{c-}(t)\right)}+e^{\lambda\left(f(t)-f_{c-}(t)\right)}\right)\right] d W(t) .
\end{aligned}
$$

I can use the definition of the hyperbolic sine and its properties (Harris and Stocker, 1998, pp. 247-249) to argue that

$$
\begin{aligned}
e^{-\lambda\left(f(t)-f_{c-}(t)\right)}-e^{\lambda\left(f(t)-f_{c-}(t)\right)} & =\frac{1}{\bar{B}} q(t)=-2 \sinh \left(\lambda\left(f(t)-f_{c-}(t)\right)\right), \\
e^{-\lambda\left(f(t)-f_{c-}(t)\right)}+e^{\lambda\left(f(t)-f_{c-}(t)\right)} & =2 \cosh \left(\lambda\left(f(t)-f_{c-}(t)\right)\right) .
\end{aligned}
$$

By the properties of $\sinh (\cdot)$ it follows that

$$
\begin{aligned}
2 \cosh \left(\lambda\left(f(t)-f_{c-}(t)\right)\right) & =\frac{\sinh \left(2\left(\lambda\left(f(t)-f_{c-}(t)\right)\right)\right)}{\sinh \left(\lambda\left(f(t)-f_{c-}(t)\right)\right)} \\
\sinh \left(2\left(\lambda\left(f(t)-f_{c-}(t)\right)\right)\right) & =2 \sinh \left(\lambda\left(f(t)-f_{c-}(t)\right)\right) \cosh \left(\lambda\left(f(t)-f_{c-}(t)\right)\right) \\
& =2 \sinh \left(\lambda\left(f(t)-f_{c-}(t)\right)\right) \sqrt[2]{1+\sinh ^{2}\left(\lambda\left(f(t)-f_{c-}(t)\right)\right)},
\end{aligned}
$$


from which I get that

$$
\begin{aligned}
e^{-\lambda\left(f(t)-f_{c-}(t)\right)}+e^{\lambda\left(f(t)-f_{c-}(t)\right)} & =2 \cosh \left(\lambda\left(f(t)-f_{c-}(t)\right)\right) \\
& =\frac{\sinh \left(2\left(\lambda\left(f(t)-f_{c-}(t)\right)\right)\right)}{\sinh \left(\lambda\left(f(t)-f_{c-}(t)\right)\right)} \\
& =2 \sqrt[2]{1+\sinh ^{2}\left(\lambda\left(f(t)-f_{c-}(t)\right)\right)} \\
& =2 \sqrt[2]{1+\frac{1}{4 \bar{B}^{2}} q(t)^{2}} .
\end{aligned}
$$

This allows me to replace the fundamentals in the dynamics of $s(t)$ and $q(t)$, i.e.,

$$
\begin{aligned}
& d s(t)=\frac{1}{2} \kappa^{2} \lambda^{2} q(t) d t+\kappa\left[1-2 \lambda \bar{B} \sqrt[2]{1+\frac{1}{4 \bar{B}^{2}} q(t)^{2}}\right] d W(t), s(0)=f_{c}(0)=0 \\
& d q(t)=\frac{1}{2} \kappa^{2} \lambda^{2} q(t) d t-2 \kappa \lambda \bar{B} \sqrt[2]{1+\frac{1}{4 \bar{B}^{2}} q(t)^{2}} d W(t), q(0)=0 .
\end{aligned}
$$

This presents us with an alternative specification for the dynamics of the nominal exchange rate.

Proof of Proposition 2. (a) From Theorem 1 in Appendix A and diffusion (5), I can characterize $k(f)$ with $\frac{1}{2} \kappa^{2} k^{\prime \prime}(f)=0$. This ODE has an explicit analytic solution, i.e.,

$$
k(f)=C_{k 1}+C_{k 2} f,
$$

for any given pair of constants $C_{k 1}$ and $C_{k 2}$. Using this result, I derive the probability $p$ of hitting the upper margin of the intervention band as

$$
\begin{aligned}
p & \equiv P_{f_{c-}(\tau)}\left(f(\tau)=f_{c-}(\tau)+\bar{f}\right) \\
& =\frac{\left(C_{k 1}+C_{k 2} f_{c-}(\tau)\right)-\left(C_{k 1}+C_{k 2}\left(f_{c-}(\tau)-\bar{f}\right)\right)}{\left(C_{k 1}+C_{k 2}\left(f_{c-}(\tau)+\bar{f}\right)\right)-\left(C_{k 1}+C_{k 2}\left(f_{c-}(\tau)-\bar{f}\right)\right)}=\frac{1}{2} .
\end{aligned}
$$

(b) From Theorem 1 in Appendix A and diffusion (5), I can characterize $h(f)$ with $\frac{1}{2} \kappa^{2} k^{\prime \prime}(f)=1$. This ODE has an explicit analytic solution, i.e.,

$$
h(f)=\left(\frac{f}{\kappa}\right)^{2}+C_{h 1}+C_{h 2} f
$$

for any given pair of constants $C_{h 1}$ and $C_{h 2}$. Using this result, I calculate the expected stopping time as

$$
\begin{aligned}
\mathbb{E}_{f_{c-}(\tau)}[\tau]= & p h\left(f_{c-}(\tau)+\bar{f}\right)+(1-p) h\left(f_{c-}(\tau)-\bar{f}\right)-h\left(f_{c-}(\tau)\right) \\
= & \frac{1}{2}\left(\left(\frac{f_{c-}(\tau)+\bar{f}}{\kappa}\right)^{2}+C_{h 1}+C_{h 2}\left(f_{c-}(\tau)+\bar{f}\right)\right)+\ldots \\
& \frac{1}{2}\left(\left(\frac{f_{c-}(\tau)-\bar{f}}{\kappa}\right)^{2}+C_{h 1}+C_{h 2}\left(f_{c-}(\tau)-\bar{f}\right)\right)-\ldots \\
& \left(\left(\frac{f_{c-}(\tau)}{\kappa}\right)^{2}+C_{h 1}+C_{h 2} f_{c-}(\tau)\right)=\left(\frac{\bar{f}}{\kappa}\right)^{2} .
\end{aligned}
$$

Hence, the expected stopping time depends on $\bar{f}$ and $\kappa$ alone. In other words, the expected stopping time depends on the width of the band $(2 \bar{f})$ and the volatility of the fundamentals $(\kappa)$. 
Proof of Proposition 3. Regardless of the realignment probability $\delta$, Proposition 2 implies that the nonsterilized interventions in the foreign exchange market $v(t)=j(t) \vec{f}$ follow a generalized random walk with variable transition probabilities and random time steps. The process $j(t)$ that counts the net number of jumps (the number of interventions that gain reserves minus the number of interventions that lose reserves) increases by one when: (a) there is an upward realignment of the band after hitting the upper margin, or (b) a defense is mounted after reaching the lower margin. The process $j(t)$ decreases by one when: (a) there is a downward realignment of the band after hitting the lower margin, or (b) a defense is mounted after reaching the upper margin. All net number of jumps $-\infty \leq j \leq \infty$ can be reached from each other with positive probability. The corresponding states of the Markov chain for the process $j(t)$ are recurrent. Hence, the unconditional distribution of the $j(t)$ process, denoted $\phi(j)$, is non-degenerate and can be computed with the following invariance recurrence:

$$
\begin{aligned}
\phi(j) & =p[\delta \phi(j+1)+(1-\delta) \phi(j-1)]+(1-p)[(1-\delta) \phi(j+1)+\delta \phi(j-1)] \\
& =\frac{1}{2} \phi(j+1)+\frac{1}{2} \phi(j-1), \text { since } p=\frac{1}{2}
\end{aligned}
$$

and the summing up constraint,

$$
\sum_{j=-\infty}^{\infty} \phi(j)=1
$$

This shows that the probability of realignment $\delta$ does not affect the unconditional distribution $\phi(j)$. The conditional distribution of net changes in foreign reserves $v(t)$ is given by $\phi(j)$ as well on re-scaled units of size $\bar{f}$ rather than one.

The size of discrete interventions to realign or defend the band is set to $\bar{f}$ units of foreign reserves. I assume that the target zone starts with $0<H<\infty$ units in foreign reserves available, that all interventions are not sterilized and that domestic policymakers alone bear the burden of managing the exchange rate. The target zone remains in place until either all foreign reserves are exhausted (drawn down to 0) or until reserves reach $N>H$ units. I abstract from the possibility of additional injections of foreign reserves after the target zone begins or from any consideration about the feasibility of sterilized operations to intervene in the foreign exchange market. After reserves hit the upper or lower bounds defined by 0 and $N$ respectively, the target zone itself is abandoned as the domestic stock of money will then fall outside an implicitly-defined desirable range.

Here I calculate the conditional probability of reaching the upper bound $N$. If domestic policymakers are willing to sustain the target zone indefinitely, then accumulation of large amounts of reserves and the concurrent increase in the money supply do not provoke a decision to abandon the target zone. As $N$ becomes arbitrarily large, I can approximate the conditional probability of a target zone that is expected to remain indefinitely. From that limit, I can calculate the probability of reaching 0 reserves and quantify the odds of depleting all available foreign reserves $H$ leading to the eventual collapse of the target zone.

$\bar{f}, H$ and $N$ are non-negative integers, so I redefine the size of any discrete intervention to be 1 unit and accordingly set $H^{\prime} \equiv \frac{H}{\bar{f}}$ and $N^{\prime} \equiv \frac{N}{\bar{f}}$. $H^{\prime}$ and $N^{\prime}$ can be interpreted as the number of net changes of size one required to accumulate $H$ and $N$ units of reserves respectively. Let me denote $\phi\left(N^{\prime} \mid H^{\prime}\right)$ the probability of accumulating $N^{\prime}$ normalized units of foreign reserves before depleting them given current holdings of $H^{\prime}$ normalized units. The conditional probability $\phi(N \mid H)$ of accumulating $N$ foreign reserves before depleting them given current holdings of $H$ units is the same as the conditional probability $\phi\left(N^{\prime} \mid H^{\prime}\right)$. The conditional probability $\phi\left(N^{\prime} \mid H^{\prime}\right)$ can be written down through the following recursion (second-order difference equation):

$$
\begin{aligned}
& (1-q) \phi\left(N^{\prime} \mid H^{\prime}+1\right)-\phi\left(N^{\prime} \mid H^{\prime}\right)+q \phi\left(N^{\prime} \mid H^{\prime}-1\right)=0 \\
& \text { where } q=p(1-\delta)+(1-p) \delta .
\end{aligned}
$$

With $\phi\left(N^{\prime} \mid H^{\prime}\right)=x^{H^{\prime}}$, I obtain the following quadratic equation:

$$
x^{2}-\frac{1}{1-q} x+\frac{q}{1-q}=0,
$$


which for any $q \neq 1$ has roots 1 and $r=\frac{q}{1-q}$. According to Proposition 2, the probability of reaching the upper margin of the intervention band is $p=\frac{1}{2}$, so it follows that $q=\frac{1}{2}$ for any probability of realignment $\delta$.

- Whenever $q \neq \frac{1}{2}$, the two characteristic roots are different from each other and the general form of the recurrence solution is a linear combination of successive powers of both roots, i.e.,

$$
\phi\left(N^{\prime} \mid H^{\prime}\right)=C_{1} 1^{H^{\prime}}+C_{2} r^{H^{\prime}}
$$

where the constants $C_{1}$ and $C_{2}$ are determined by these two boundary conditions: $\phi\left(N^{\prime} \mid N^{\prime}\right)=1$ and $\phi\left(N^{\prime} \mid 0\right)=0$. The former boundary condition indicates that the probability of reaching $N^{\prime}$ normalized units of reserves starting with $N^{\prime}$ units is trivially 1 . The latter condition simply says that starting with no reserves, the target zone collapses and hence there is no chance for reserves to increase up to $N^{\prime}$ normalized units. Hence, it follows that

$$
\left.\begin{array}{l}
0=C_{1}+C_{2} \\
1=C_{1}+C_{2} r^{N^{\prime}}
\end{array}\right\} \Rightarrow \begin{aligned}
& C_{1}=\frac{1}{1-r^{N^{\prime}}} \\
& C_{2}=\frac{-1}{1-r^{N^{\prime}}}
\end{aligned}
$$

so the probability of reaching $N^{\prime}$ normalized units of reserves before the collapse of the target zone is

$$
\phi\left(N^{\prime} \mid H^{\prime}\right)=\frac{1-r^{H^{\prime}}}{1-r^{N^{\prime}}} .
$$

- Whenever $q=\frac{1}{2}$, I obtain the solution that is applicable to the target zone model as implied by Proposition 2. In that case, the two characteristic roots are equal and the general form of the solution to the recursion is

$$
\phi\left(N^{\prime} \mid H^{\prime}\right)=C_{1}+C_{2} H^{\prime} .
$$

Given the same boundary conditions as before, $C_{1}=0$ and $C_{2}=\frac{1}{N^{\prime}}$, and the solution becomes

$$
\phi\left(N^{\prime} \mid H^{\prime}\right)=\frac{H^{\prime}}{N^{\prime}} .
$$

○ Since $\phi\left(N^{\prime} \mid H^{\prime}\right)=\phi(N \mid H)$, it follows that

$$
\phi(N \mid H)=\left\{\begin{array}{c}
\frac{1-r^{\frac{H}{f}}}{1-r^{\frac{N}{f}}} \text { if } q \neq \frac{1}{2} \\
\frac{H}{N} \text { if } q=\frac{1}{2}
\end{array}\right.
$$

is the probability of reaching $N$ units of reserves before the target zone collapses with discrete interventions of size $\bar{f}$. For any discrete intervention size $\bar{f}$ and any initial holdings $H$, I approximate the probability of eventual collapse whenever the target zone is set to remain indefinitely as 1 minus the limit of the conditional probability $\phi(N \mid H)$ as $N$ becomes arbitrarily large, i.e.,

$$
1-\lim _{N \rightarrow \infty} \phi(N \mid H)=\left\{\begin{array}{l}
1 \text { if } q \geq \frac{1}{2} \\
1 \text { if } q=\frac{1}{2} \\
r^{\frac{H}{f}}=\left(\frac{q}{1-q}\right)^{\frac{H}{f}} \text { if } q<\frac{1}{2} .
\end{array}\right.
$$

In other words, the probability of an eventual collapse of the target zone approaches 1 if the probability of losing reserves conditional on hitting the band satisfies that $q \geq \frac{1}{2}$, irrespective of the probability of realignment $\delta$. The probability of eventual collapse whenever $q<\frac{1}{2}$ is determined by the initial reserves $H$, the size of the discrete intervention $\bar{f}$, and the probability $q=p(1-\delta)+(1-p) \delta$ itself.

Given that the probability of realignment $\delta$ does not have an effect on $q$ for the model described in the paper (since, according to Proposition 2, the probability of reaching the upper margin of the intervention band is $p=\frac{1}{2}$ so $q=\frac{1}{2}$ ), the implication is that parity realignments do not alter the nature of the gambler's ruin problem faced by an exchange rate target zone with imperfect credibility. 


\section{An Illustration}

In Figure 1 below, I illustrate the exchange rate solution in a free-floating regime (red solid line). I also show the inverted "S-curve" that arises within a target zone with low credibility (blue solid line) and the "S-curve" characteristic of a high credibility target zone (green solid line). The parameters used for this plot are $\beta=0.99, \gamma=1$ and $\kappa=0.071066$ which all together imply that $\lambda=\sqrt[2]{2 \frac{\gamma}{\kappa^{2}}\left(\frac{1-\beta}{\beta}\right)}=2$. I set the upper band on fundamentals to $\bar{f}=1$ and the midpoint at the zero-origin (i.e., $\left.f_{c-}(0)=f_{c}(0)=0\right)$. Then, I choose $\delta=0.75$ for the low credibility case (implying that $B \equiv-e^{\lambda f_{c-}(0)}\left(\delta-\frac{1}{2}\right) \frac{\bar{f}}{\sinh (\lambda \bar{f})}=-0.06893$ ), and $\delta=0.25$ for the high credibility case (implying that $\left.B \equiv-e^{\lambda f_{c-}(0)}\left(\delta-\frac{1}{2}\right) \frac{\bar{f}}{\sinh (\lambda \bar{f})}=0.06893\right) . B=0$ defines the free-floating solution.

In the framework with imperfectly credible target zones and discrete interventions, a given symmetric band on fundamentals $[f, \bar{f}]$ where $\underline{f}=-\bar{f}$ leads to wider bands on the exchange rate $[\underline{s}, \bar{s}]$ for increasingly less credible target zones. As noted in the paper, the margins on the exchange rate are related to the width of the margins of intervention on fundamentals $(2 \bar{f})$ and the probability of realignment $(\delta)$ as follows: $\bar{s}=2 \delta \bar{f}$, and $\underline{s}=-\bar{s}$. For the illustration in Figure 1, the margins on the exchange rate for the low credibility case $(\delta=0.75)$ become $[\underline{s}, \bar{s}]=[-1.5,1.5]$ while for the high credibility case $(\delta=0.25)$ are $[\underline{s}, \bar{s}]=[-0.5,0.5]$.

If two target zones differ on the probability of realignment $\delta$ but impose the same margins on the exchange rate $[\underline{s}, \bar{s}]$, then it has to be the case that the less credible target zone has a tighter intervention band defined over the fundamentals than the more credible one. Alternatively, if the width of the intervention band on fundamentals is the same, then the width of the target zone over the nominal exchange rate must be larger for less credible target zones - that, in turn, reveals the differences in credibility between both exchange rate arrangements. 
Figure 1. The Exchange Rate as a Function of Economic Fundamentals: Free-floating vs. Target Zone with Imperfect Credibility

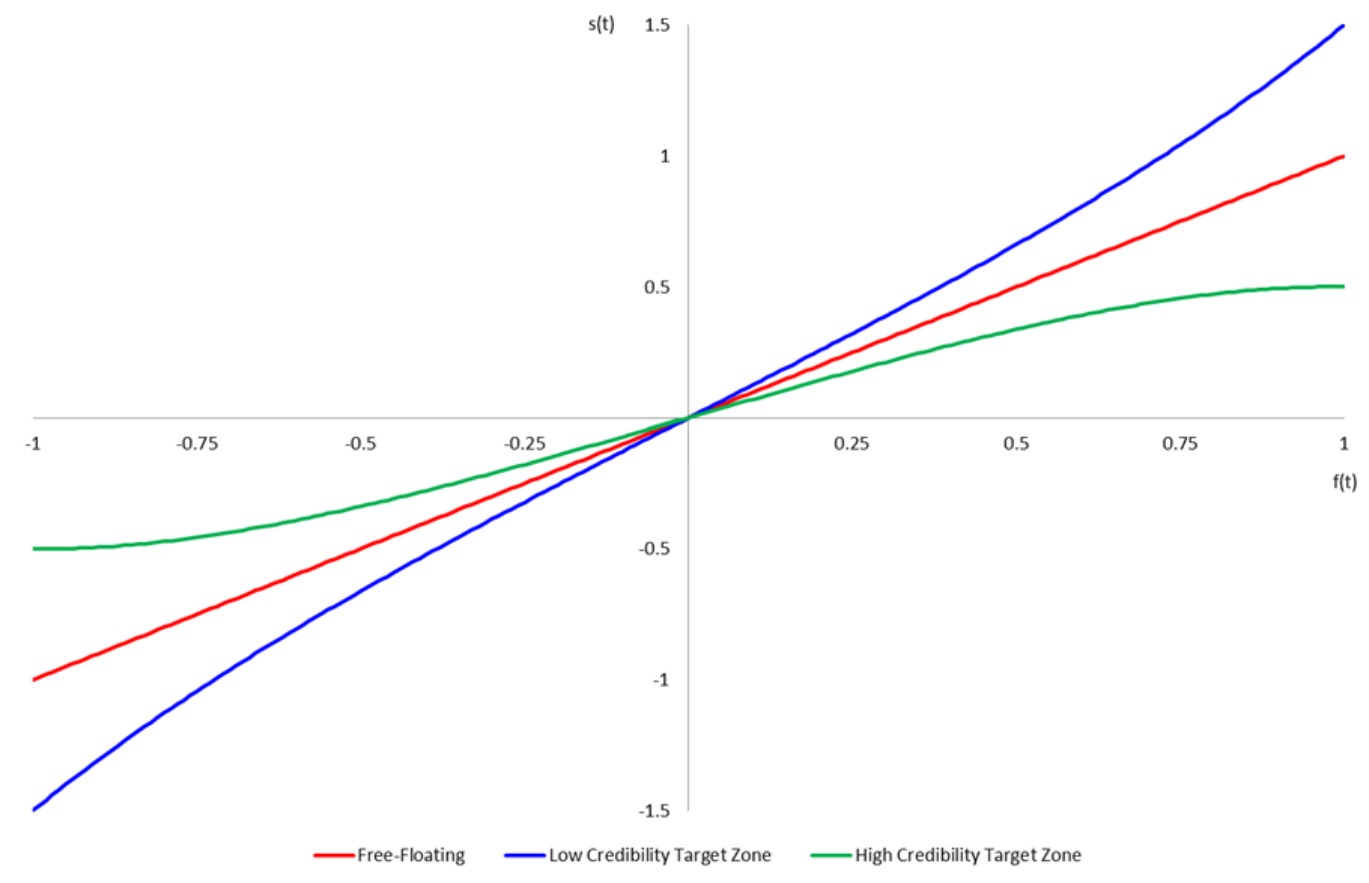

Figure 1: The predictions for the exchange rate under a free-floating regime are represented by the red line. The "S-curve" prediction for a target zone with high credibility is represented by the green line. The inverted "S-curve" prediction for a target zone with low credibility is represented by the blue line. 